IJMMS 27:1 (2001) 7-15

PII. S0161171201010419

http://ijmms.hindawi.com

(c) Hindawi Publishing Corp.

\title{
ON AN APPLICATION OF ALMOST INCREASING SEQUENCES
}

\section{HÜSEYIN BOR}

(Received 3 May 2000 and in revised form 25 October 2000)

\begin{abstract}
Using an almost increasing sequence, a result of Mazhar (1977) on $|C, 1|_{k}$ summability factors has been generalized for $|C, \alpha ; \beta|_{k}$ and $\left|\bar{N}, p_{n} ; \beta\right|_{k}$ summability factors under weaker conditions.
\end{abstract}

2000 Mathematics Subject Classification. 40D15, 40F05, 40G05.

1. Introduction. A sequence of $\left(b_{n}\right)$ of positive numbers is said to be $\delta$-quasimonotone, if $b_{n} \rightarrow 0, b_{n}>0$ ultimately and $\Delta b_{n} \geq-\delta_{n}$, where $\left(\delta_{n}\right)$ is a sequence of positive numbers (see [2]). Let $\sum a_{n}$ be a given infinite series with $\left(s_{n}\right)$ as the sequence of its $n$th partial sums. Let $\sigma_{n}^{\alpha}$ and $t_{n}^{\alpha}$ denote the $n$th $(C, \alpha)$ means of the sequences $\left(s_{n}\right)$ and $\left(n a_{n}\right)$, respectively, that is,

$$
\begin{gathered}
\sigma_{n}^{\alpha}=\frac{1}{A_{n}^{\alpha}} \sum_{v=0}^{n} A_{n-v}^{\alpha-1} s_{v}, \\
t_{n}^{\alpha}=\frac{1}{A_{n}^{\alpha}} \sum_{v=1}^{n} A_{n-v}^{\alpha-1} v a_{v},
\end{gathered}
$$

where

$$
A_{n}^{\alpha}=O\left(n^{\alpha}\right), \quad \alpha>-1, \quad A_{0}^{\alpha}=1, \quad A_{-n}^{\alpha}=0, \quad \text { for } n>0 .
$$

The series $\sum a_{n}$ is said to be summable $|C, \alpha|_{k}, k \geq 1$ and $\alpha>-1$, if (see [6])

$$
\sum_{n=1}^{\infty} n^{k-1}\left|\sigma_{n}^{\alpha}-\sigma_{n-1}^{\alpha}\right|^{k}=\sum_{n=1}^{\infty} \frac{1}{n}\left|t_{n}^{\alpha}\right|^{k}<\infty,
$$

and it is said to be summable $|C, \alpha ; \beta|_{k}, k \geq 1, \alpha>-1$ and $\beta \geq 0$, if (see [7])

$$
\sum_{n=1}^{\infty} n^{\beta k+k-1}\left|\sigma_{n}^{\alpha}-\sigma_{n-1}^{\alpha}\right|^{k}=\sum_{n=1}^{\infty} n^{\beta k-1}\left|t_{n}^{\alpha}\right|^{k}<\infty .
$$

Let $\left(p_{n}\right)$ be a sequence of positive numbers such that

$$
P_{n}=\sum_{v=0}^{n} p_{v} \rightarrow \infty \quad \text { as } n \longrightarrow \infty, P_{-i}=p_{-i}=0, i \geq 1 .
$$

The sequence-to-sequence transformation

$$
T_{n}=\frac{1}{P_{n}} \sum_{v=0}^{n} p_{v} s_{v}
$$


defines the sequence $\left(T_{n}\right)$ of the Riesz mean or simply the $\left(\bar{N}, p_{n}\right)$ mean of the sequence $\left(s_{n}\right)$, generated by the sequence of coefficients $\left(p_{n}\right)$ (see [8]).

The series $\sum a_{n}$ is said to be summable $\left|\bar{N}, p_{n}\right|_{k}, k \geq 1$, if (see [3])

$$
\sum_{n=1}^{\infty}\left(\frac{P_{n}}{p_{n}}\right)^{k-1}\left|\Delta T_{n-1}\right|^{k}<\infty
$$

and it is said to be summable $\left|\bar{N}, p_{n} ; \beta\right|_{k}, k \geq 1$, and $\beta \geq 0$, if (see [4])

$$
\sum_{n=1}^{\infty}\left(\frac{P_{n}}{p_{n}}\right)^{\beta k+k-1}\left|\Delta T_{n-1}\right|^{k}<\infty
$$

where

$$
\Delta T_{n-1}=-\frac{p_{n}}{P_{n} P_{n-1}} \sum_{v=1}^{n} P_{v-1} a_{v}, \quad n \geq 1
$$

In the special case when $\beta=0$ (resp., $p_{n}=1$ for all values of $n$ ), $\left|\bar{N}, p_{n} ; \beta\right|_{k}$ summability is the same as $\left|\bar{N}, p_{n}\right|_{k}$ (resp., $|C, 1 ; \beta|_{k}$ ) summability.

Also it is known that $|C, \alpha ; \beta|_{k}$ and $\left|\bar{N}, p_{n} ; \beta\right|_{k}$ summabilities are, in general, independent of each other.

Mazhar [9] has proved the following theorem for $|C, 1|_{k}$ summability factors of infinite series.

THEOREM 1.1 (see [9]). Let $\lambda_{n} \rightarrow 0$ as $n \rightarrow \infty$. Suppose that there exists a sequence of numbers $\left(B_{n}\right)$ such that it is $\delta$-quasi-monotone with $\sum n \delta_{n} \log n<\infty, \sum B_{n} \log n$ is convergent and $\left|\Delta \lambda_{n}\right| \leq\left|B_{n}\right|$ for all $n$. If

$$
\sum_{n=1}^{m} \frac{1}{n}\left|t_{n}\right|^{k}=O(\log m) \quad \text { as } m \longrightarrow \infty,
$$

where $\left(t_{n}\right)$ is the $n t h(C, 1)$ mean of the sequence $\left(n a_{n}\right)$, then the series $\sum a_{n} \lambda_{n}$ is summable $|C, 1|_{k}, k \geq 1$.

REMARK 1.2. It should be noted that the condition " $\sum n B_{n} \log n$ is convergent" is enough to prove Theorem 1.1 rather than the conditions " $\sum n \delta_{n} \log n<\infty$ and $\sum B_{n} \log n$ is convergent."

2. The main result. In view of Remark 1.2, the aim of this paper is to generalize Theorem 1.1 for $|C, \alpha ; \beta|_{k}$ and $\left|\bar{N}, p_{n} ; \beta\right|_{k}$ summabilities under weaker conditions. For this we need the concept of almost increasing sequence. A positive sequence $\left(d_{n}\right)$ is said to be almost increasing if there exists a positive increasing sequence $\left(c_{n}\right)$ and two positive constants $A$ and $B$ such that $A c_{n} \leq d_{n} \leq B c_{n}$ (see [1]). Obviously, every increasing sequence is almost increasing but the converse need not be true as can be seen from the example $d_{n}=n e^{(-1)^{n}}$. Since $\log n$ is increasing, we are weakening the hypotheses of the theorem replacing the increasing sequence by an almost increasing sequence. 
Now, we prove the following theorems.

TheOREM 2.1. Let $\left(X_{n}\right)$ be an almost increasing sequence and $\lambda_{n} \rightarrow 0$ as $n \rightarrow \infty$. Suppose that there exists a sequence of numbers $\left(B_{n}\right)$ such that it is $\delta$-quasi-monotone with $\sum n B_{n} X_{n}$ convergent and $\left|\Delta \lambda_{n}\right| \leq\left|B_{n}\right|$ for all $n$. If the sequence $\left(u_{n}^{\alpha}\right)$, defined by (see [10])

$$
u_{n}^{\alpha}= \begin{cases}\left|t_{n}^{\alpha}\right|, & \alpha=1, \\ \max _{1 \leq v \leq n}\left|t_{v}^{\alpha}\right|, & 0<\alpha<1,\end{cases}
$$

satisfies the condition

$$
\sum_{n=1}^{m} n^{\beta k-1}\left(u_{n}^{\alpha}\right)^{k}=O\left(X_{m}\right) \quad \text { as } m \longrightarrow \infty,
$$

then the series $\sum a_{n} \lambda_{n}$ is summable $|C, \alpha ; \beta|_{k}, k \geq 1$ and $0 \leq \beta<\alpha \leq 1$.

THEOREM 2.2. Let $\left(X_{n}\right)$ be an almost increasing sequence and $\lambda_{n} \rightarrow 0$ as $n \rightarrow \infty$. Suppose that there exists a sequence of numbers $\left(B_{n}\right)$ such that it is $\delta$-quasi-monotone with $\sum n B_{n} X_{n}$ convergent and $\left|\Delta \lambda_{n}\right| \leq\left|B_{n}\right|$ for all $n$. If $\left(p_{n}\right)$ is a sequence such that

$$
\begin{aligned}
& \sum_{n=v+1}^{\infty}\left(\frac{P_{n}}{p_{n}}\right)^{\beta k-1} \frac{1}{P_{n-1}}=O\left\{\left(\frac{P_{v}}{p_{v}}\right)^{\beta k} \frac{1}{P_{v}}\right\}, \\
& \sum_{n=1}^{m}\left(\frac{P_{n}}{p_{n}}\right)^{\beta k-1}\left|t_{n}\right|^{k}=O\left(X_{m}\right) \text { as } m \rightarrow \infty, \\
& \sum_{n=1}^{m}\left(\frac{P_{n}}{p_{n}}\right)^{\beta k} \frac{1}{n}\left|t_{n}\right|^{k}=O\left(X_{m}\right) \quad \text { as } m \longrightarrow \infty, \\
& \sum_{n=1}^{m} \frac{\left|\lambda_{n}\right|}{n}=O(1) \quad \text { as } m \rightarrow \infty,
\end{aligned}
$$

then the series $\sum a_{n} \lambda_{n}$ is summable $\left|\bar{N}, p_{n} ; \beta\right|_{k}$ for $k \geq 1$ and $0 \leq \beta<1 / k$.

We need the following lemmas for the proof of our theorems.

LEMMA 2.3 (see [5]). If $0<\alpha \leq 1$ and $1 \leq v \leq n$, then

$$
\left|\sum_{p=0}^{v} A_{n-p}^{\alpha-1} a_{p}\right| \leq \max _{1 \leq m \leq v}\left|\sum_{p=0}^{m} A_{m-p}^{\alpha-1} a_{p}\right| .
$$

Under the conditions of Theorem 2.2 we obtain the following result.

LEMMA 2.4. The following equation holds:

$$
\left|\lambda_{n}\right| X_{n}=O(1) \text { as } n \rightarrow \infty \text {. }
$$


Proof. Since $\lambda_{n} \rightarrow 0$ as $n \rightarrow \infty$, we have

$$
\left|\lambda_{n}\right| X_{n}=X_{n}\left|\sum_{v=n}^{\infty} \Delta \lambda_{v}\right| \leq X_{n} \sum_{v=n}^{\infty}\left|\Delta \lambda_{v}\right| \leq \sum_{v=0}^{\infty} X_{v}\left|\Delta \lambda_{v}\right| \leq \sum_{v=0}^{\infty} X_{v}\left|B_{v}\right|<\infty .
$$

Hence $\left|\lambda_{n}\right| X_{n}=O(1)$ as $n \rightarrow \infty$.

3. Proof of Theorem 2.1. Let $\left(T_{n}^{\alpha}\right)$ be the $n$th $(C, \alpha)$, with $0<\alpha \leq 1$, mean of the sequence $\left(n a_{n} \lambda_{n}\right)$. Then, by (1.1), we have

$$
T_{n}^{\alpha}=\frac{1}{A_{n}^{\alpha}} \sum_{v=1}^{n} A_{n-v}^{\alpha-1} v a_{v} \lambda_{v}
$$

Applying Abel's transformation, we get

$$
T_{n}^{\alpha}=\frac{1}{A_{n}^{\alpha}} \sum_{v=1}^{n-1} \Delta \lambda_{v} \sum_{p=1}^{v} A_{n-p}^{\alpha-1} p a_{p}+\frac{\lambda_{n}}{A_{n}^{\alpha}} \sum_{v=1}^{n} A_{n-v}^{\alpha-1} v a_{v}
$$

so that making use of Lemma 2.3, we have

$$
\begin{aligned}
\left|T_{n}^{\alpha}\right| & \leq \frac{1}{A_{n}^{\alpha}} \sum_{v=1}^{n-1}\left|\Delta \lambda_{v}\right|\left|\sum_{p=1}^{v} A_{n-p}^{\alpha-1} p a_{p}\right|+\frac{\left|\lambda_{n}\right|}{A_{n}^{\alpha}}\left|\sum_{v=1}^{n} A_{n-v}^{\alpha-1} v a_{v}\right| \\
& \leq \frac{1}{A_{n}^{\alpha}} \sum_{v=1}^{n-1} A_{v}^{\alpha} u_{v}^{\alpha}\left|\Delta \lambda_{v}\right|+\left|\lambda_{n}\right| u_{n}^{\alpha} \\
& =T_{n, 1}^{\alpha}+T_{n, 2}^{\alpha} .
\end{aligned}
$$

Since

$$
\left|T_{n, 1}^{\alpha}+T_{n, 2}^{\alpha}\right|^{k} \leq 2^{k}\left(\left|T_{n, 1}^{\alpha}\right|^{k}+\left|T_{n, 2}^{\alpha}\right|^{k}\right)
$$

to complete the proof of Theorem 2.1, it is enough to show that

$$
\sum_{n=1}^{\infty} n^{\beta k-1}\left|T_{n, r}^{\alpha}\right|^{k}<\infty \quad \text { for } r=1,2
$$

Now, when $k>1$, applying Hölder's inequality with indices $k$ and $k^{\prime}$, where $1 / k+1 / k^{\prime}=$ 1 , we get

$$
\begin{aligned}
& \sum_{n=2}^{m+1} n^{\beta k-1}\left|T_{n, 1}^{\alpha}\right|^{k} \\
& \quad \leq \sum_{n=2}^{m+1} n^{\beta k-1}\left(A_{n}^{\alpha}\right)^{-k}\left\{\sum_{v=1}^{n-} A_{v}^{\alpha} u_{v}^{\alpha}\left|B_{v}\right|\right\}^{k}
\end{aligned}
$$


ON AN APPLICATION OF ALMOST INCREASING SEQUENCES

$$
\begin{aligned}
& \leq \sum_{n=2}^{m+1} n^{\beta k-1}\left(A_{n}^{\alpha}\right)^{-k}\left\{\sum_{v=1}^{n-1}\left(A_{v}^{\alpha}\right)^{k}\left(u_{v}^{\alpha}\right)^{k}\left|B_{v}\right|\right\}\left\{\sum_{v=1}^{n-1}\left|B_{v}\right|\right\}^{k-1} \\
& =O(1) \sum_{n=2}^{m+1} n^{\beta k-\alpha k-1}\left\{\sum_{v=1}^{n-1} v^{\alpha k}\left(u_{v}^{\alpha}\right)^{k}\left|B_{v}\right|\right\} \\
& =O(1) \sum_{v=1}^{m} v^{\alpha k}\left(u_{v}^{\alpha}\right)^{k}\left|B_{v}\right| \sum_{n=v+1}^{m+1} \frac{1}{n^{1+\alpha k-\beta k}} \\
& =O(1) \sum_{v=1}^{m} v^{\alpha k}\left(u_{v}^{\alpha}\right)^{k}\left|B_{v}\right| \int_{v}^{\infty} \frac{d x}{x^{1+\alpha k-\beta k}} \\
& =O(1) \sum_{v=1}^{m} v^{\beta k}\left(u_{v}^{\alpha}\right)^{k}\left|B_{v}\right|=O(1) \sum_{v=1}^{m} v\left|B_{v}\right| v^{\beta k-1}\left(u_{v}^{\alpha}\right)^{k} \\
& =O(1) \sum_{v=1}^{m-1} \Delta\left(v\left|B_{v}\right|\right) \sum_{r=1}^{v} r^{\beta k-1}\left(u_{r}^{\alpha}\right)^{k}+O(1) m\left|B_{m}\right| \sum_{v=1}^{m} v^{\beta k-1}\left(u_{v}^{\alpha}\right)^{k} \\
& =O(1) \sum_{v=1}^{m-1}\left|\Delta\left(v\left|B_{v}\right|\right)\right| X_{v}+O(1) m\left|B_{m}\right| X_{m} \\
& =O(1) \sum_{v=1}^{m-1} v\left|B_{v}\right| X_{v}+O(1) \sum_{v=1}^{m-1}(v+1)\left|B_{v+1}\right| X_{v+1}+O(1) m\left|B_{m}\right| X_{m} \\
& =O(1) \quad \text { as } m \rightarrow \infty,
\end{aligned}
$$

by virtue of the hypotheses of Theorem 2.1.

Finally, since $\left|\lambda_{n}\right|=O(1)$, by hypothesis

$$
\begin{aligned}
\sum_{n=1}^{m} n^{\beta k-1}\left|T_{n, 2}^{\alpha}\right|^{k} & =\sum_{n=1}^{m}\left|\lambda_{n}\right|^{k-1} n^{\beta k-1}\left(u_{n}^{\alpha}\right)^{k} \\
& =O(1) \sum_{n=1}^{m}\left|\lambda_{n}\right| n^{\beta k-1}\left(u_{n}^{\alpha}\right)^{k} \sum_{v=n}^{\infty}\left|\Delta \lambda_{v}\right| \\
& =O(1) \sum_{v=1}^{\infty}\left|\Delta \lambda_{v}\right| \sum_{n=1}^{v} n^{\beta k-1}\left(u_{v}^{\alpha}\right)^{k} \\
& =O(1) \sum_{v=1}^{\infty}\left|B_{v}\right| X_{v}<\infty,
\end{aligned}
$$

by virtue of the hypotheses of Theorem 2.1.

Therefore, we get

$$
\sum_{n=1}^{m} n^{\beta k-1}\left|T_{n, r}^{\alpha}\right|^{k}=O(1) \quad \text { as } m \longrightarrow \infty, \text { for } r=1,2 .
$$

This completes the proof of Theorem 2.1.

REMARK 3.1. It is natural to ask whether our theorem is true with $\alpha>1$. All we can say with certainty is that our proof fails if $\alpha>1$, for our estimate of $T_{n, 1}^{\alpha}$ depends upon Lemma 2.3, and Lemma 2.3 is known to be false when $\alpha>1$ (see [5] for details). 
Proof of TheOrem 2.2. Let $\left(T_{n}\right)$ denotes the $\left(\bar{N}, p_{n}\right)$ mean of the series $\sum a_{n} \lambda_{n}$. Then, by definition and changing the order of summation, we have

$$
T_{n}=\frac{1}{P_{n}} \sum_{v=0}^{n} p_{v} \sum_{i=0}^{v} a_{i} \lambda_{i}=\frac{1}{P_{n}} \sum_{v=0}^{n}\left(P_{n}-P_{v-1}\right) a_{v} \lambda_{v} .
$$

Then, for $n \geq 1$, we have

$$
T_{n}-T_{n-1}=\frac{p_{n}}{P_{n} P_{n-1}} \sum_{v=1}^{n} P_{v-1} a_{v} \lambda_{v}=\frac{p_{n}}{P_{n} P_{n-1}} \sum_{v=1}^{n} \frac{P_{v-1} \lambda_{v}}{v} v a_{v}
$$

By Abel's transformation, we have

$$
\begin{aligned}
T_{n}-T_{n-1}= & \frac{n+1}{n P_{n}} p_{n} t_{n} \lambda_{n}-\frac{p_{n}}{P_{n} P_{n-1}} \sum_{v=1}^{n-1} p_{v} t_{v} \lambda_{v} \frac{v+1}{v} \\
& +\frac{p_{n}}{P_{n} P_{n-1}} \sum_{v=1}^{n-1} P_{v} \Delta \lambda_{v} t_{v} \frac{v+1}{v}+\frac{p_{n}}{P_{n} P_{n-1}} \sum_{v=1}^{n-1} P_{v} t_{v} \lambda_{v+1} \frac{1}{v} \\
= & T_{n, 1}+T_{n, 2}+T_{n, 3}+T_{n, 4} .
\end{aligned}
$$

Since

$$
\left|T_{n, 1}+T_{n, 2}+T_{n, 3}+T_{n, 4}\right|^{k} \leq 4^{k}\left(\left|T_{n, 1}\right|^{k}+\left|T_{n, 2}\right|^{k}+\left|T_{n, 3}\right|^{k}+\left|T_{n, 4}\right|^{k}\right),
$$

to complete the proof of Theorem 2.2, it is enough to show that

$$
\sum_{n=1}^{\infty}\left(\frac{P_{n}}{p_{n}}\right)^{\beta k+k-1}\left|T_{n, r}\right|^{k}<\infty \text { for } r=1,2,3,4 .
$$

Since $\left(\lambda_{n}\right) \rightarrow 0$ as $n \rightarrow \infty$ by the hypothesis of Theorem 2.2, we have

$$
\begin{aligned}
\sum_{n=1}^{m}\left(\frac{P_{n}}{p_{n}}\right)^{\beta k+k-1}\left|T_{n, 1}\right|^{k}= & O(1) \sum_{n=1}^{m}\left(\frac{P_{n}}{p_{n}}\right)^{\beta k-1}\left|\lambda_{n}\right|^{k-1}\left|\lambda_{n}\right|\left|t_{n}\right|^{k} \\
= & O(1) \sum_{n=1}^{m}\left|\lambda_{n}\right|\left(\frac{P_{n}}{p_{n}}\right)^{\beta k-1}\left|t_{n}\right|^{k} \\
= & O(1) \sum_{n=1}^{m-1} \Delta\left|\lambda_{n}\right| \sum_{v=1}^{n}\left(\frac{P_{v}}{p_{v}}\right)^{\beta k-1}\left|t_{v}\right|^{k} \\
& +O(1)\left|\lambda_{m}\right| \sum_{n=1}^{m}\left(\frac{P_{n}}{p_{n}}\right)^{\beta k-1}\left|t_{n}\right|^{k} \\
= & O(1) \sum_{n=1}^{m-1}\left|\Delta \lambda_{n}\right| X_{n}+O(1)\left|\lambda_{m}\right| X_{m} \\
= & O(1) \sum_{n=1}^{m-1}\left|B_{n}\right| X_{n}+O(1)\left|\lambda_{m}\right| X_{m}=O(1) \quad \text { as } m \longrightarrow \infty,
\end{aligned}
$$

by virtue of the hypotheses of Theorem 2.2 and in view of Lemma 2.4. 
Now, when $k>1$, applying Hölder's inequality with indices $k$ and $k^{\prime}$, where $1 / k+$ $1 / k^{\prime}=1$, as in $T_{n, 1}$, we have

$$
\begin{aligned}
\sum_{n=2}^{m+1}\left(\frac{P_{n}}{p_{n}}\right)^{\beta k+k-1}\left|T_{n, 2}\right|^{k}= & O(1) \sum_{n=2}^{m+1}\left(\frac{P_{n}}{p_{n}}\right)^{\beta k-1} \frac{1}{P_{n-1}}\left\{\sum_{v=1}^{n-1} p_{v}\left|\lambda_{v}\right|^{k}\left|t_{v}\right|^{k}\right\} \\
& \times\left\{\frac{1}{P_{n-1}} \sum_{v=1}^{n-1} p_{v}\right\}^{k-1} \\
= & O(1) \sum_{v=1}^{m} p_{v}\left|\lambda_{v}\right|^{k-1}\left|\lambda_{v}\right|\left|t_{v}\right|^{k} \sum_{n=v+1}^{m+1}\left(\frac{P_{n}}{p_{n}}\right)^{\beta k-1} \frac{1}{P_{n-1}} \\
= & O(1) \sum_{v=1}^{m}\left(\frac{P_{v}}{p_{v}}\right)^{\beta k-1}\left|t_{v}\right|^{k}\left|\lambda_{v}\right|=O(1) \quad \text { as } m \rightarrow \infty
\end{aligned}
$$

Again, we have

$$
\begin{aligned}
\sum_{n=2}^{m+1}\left(\frac{P_{n}}{p_{n}}\right)^{\beta k+k-1}\left|T_{n, 3}\right|^{k}= & O(1) \sum_{n=2}^{m+1}\left(\frac{P_{n}}{p_{n}}\right)^{\beta k-1} \frac{1}{P_{n-1}}\left\{\sum_{v=1}^{n-1} P_{v}\left|B_{v}\right|\left|t_{v}\right|^{k}\right\} \\
& \times\left\{\frac{1}{P_{n-1}} \sum_{v=1}^{n-1} P_{v}\left|B_{v}\right|\right\}^{k-1} \\
= & O(1) \sum_{v=1}^{m} P_{v}\left|B_{v}\right|\left|t_{v}\right|^{k} \sum_{n=v+1}^{m+1}\left(\frac{P_{n}}{p_{n}}\right)^{\beta k-1} \frac{1}{P_{n-1}} \\
= & O(1) \sum_{v=1}^{m}\left|B_{v}\right|\left(\frac{P_{v}}{p_{v}}\right)^{\beta k}\left|t_{v}\right|^{k} \\
= & O(1) \sum_{v=1}^{m} v\left|B_{v}\right|\left(\frac{P_{v}}{p_{v}}\right)^{\beta k} \frac{1}{v}\left|t_{v}\right|^{k} \\
= & O(1) \sum_{v=1}^{m-1} \Delta\left(v\left|B_{v}\right|\right) \sum_{i=1}^{v}\left(\frac{P_{i}}{p_{i}}\right)^{\beta k} \frac{1}{i}\left|t_{i}\right|^{k} \\
& +O(1) m\left|B_{m}\right| \sum_{v=1}^{m}\left(\frac{P_{v}}{p_{v}}\right)^{\beta k} \frac{1}{v}\left|t_{v}\right|^{k} \\
= & O(1) \sum_{v=1}^{m-1}\left|\Delta\left(v\left|B_{v}\right|\right)\right| X_{v}+O(1) m\left|B_{m}\right| X_{m} \\
= & O(1) \sum_{v=1}^{m-1} v X_{v}\left|B_{v}\right|+O(1) \sum_{v=1}^{m-1}(v+1)\left|B_{v+1}\right| X_{v+1} \\
& +O(1) m\left|B_{m}\right| X_{m} \\
= & O(1){ }^{k} \operatorname{as} \rightarrow \infty,
\end{aligned}
$$

by virtue of the hypotheses of Theorem 2.2. 
Finally, we have

$$
\begin{aligned}
\sum_{n=2}^{m+1}\left(\frac{P_{n}}{p_{n}}\right)^{\beta k+k-1}\left|T_{n, 4}\right|^{k}= & O(1) \sum_{n=2}^{m+1}\left(\frac{P_{n}}{p_{n}}\right)^{\beta k-1} \frac{1}{P_{n-1}} \sum_{v=1}^{n-1} P_{v} \frac{\left|\lambda_{v+1}\right|}{v}\left|t_{v}\right|^{k} \\
& \times\left\{\frac{1}{P_{n-1}} \sum_{v=1}^{n-1} P_{v} \frac{\left|\lambda_{v+1}\right|}{v}\right\}^{k-1} \\
= & O(1) \sum_{v=1}^{m} P_{v} \frac{\left|\lambda_{v+1}\right|}{v}\left|t_{v}\right|^{k} \sum_{n=v+1}^{m+1}\left(\frac{P_{n}}{p_{n}}\right)^{\beta k-1} \frac{1}{P_{n-1}} \\
= & O(1) \sum_{v=1}^{m}\left|\lambda_{v+1}\right|\left(\frac{P_{v}}{p_{v}}\right)^{\beta k} \frac{1}{v}\left|t_{v}\right|^{k} \\
= & O(1) \sum_{v=1}^{m-1} \Delta\left|\lambda_{v+1}\right| \sum_{r=1}^{v}\left(\frac{P_{r}}{p_{r}}\right)^{\beta k} \frac{1}{r}\left|t_{r}\right|^{k} \\
& +O(1)\left|\lambda_{m+1}\right| \sum_{v=1}^{m}\left(\frac{P_{v}}{p_{v}}\right)^{\beta k} \frac{1}{v}\left|t_{v}\right|^{k} \\
= & O(1) \sum_{v=1}^{m-1}\left|\Delta \lambda_{v+1}\right| X_{v+1}+O(1)\left|\lambda_{m+1}\right| X_{m+1} \\
= & O(1) \sum_{v=1}^{m-1}\left|B_{v+1}\right| X_{v+1}+O(1)\left|\lambda_{m+1}\right| X_{m+1} \\
= & O(1) \operatorname{as}^{m} m \infty
\end{aligned}
$$

by virtue of the hypotheses of Theorem 2.2 and in view of Lemma 2.4.

Therefore, we get

$$
\sum_{n=1}^{m}\left(\frac{P_{n}}{p_{n}}\right)^{\beta k+k-1}\left|T_{n, r}\right|^{k}=O(1) \quad \text { as } m \longrightarrow \infty, \text { for } r=1,2,3,4 .
$$

This completes the proof of Theorem 2.2.

If we take $p_{n}=1$ for all values of $n$ in this theorem, then we get a result concerning the $|C, 1 ; \beta|_{k}$ summability factors.

\section{REFERENCES}

[1] S. Aljančić and D. Arandelović, 0-regularly varying functions, Publ. Inst. Math. (Beograd) (N.S.) 22(36) (1977), 5-22. MR 57\#6317. Zbl 379.26003.

[2] R. P. Boas, Jr., Quasi-positive sequences and trigonometric series, Proc. London Math. Soc. (3) 14a (1965), 38-46. MR 31\#2556. Zbl 128.29302.

[3] H. Bor, On two summability methods, Math. Proc. Cambridge Philos. Soc. 97 (1985), no. 1, 147-149. MR 86d:40004. Zbl 554.40008.

[4] _ On local property of $\left|\bar{N}, p_{n} ; \delta\right|_{k}$ summability of factored Fourier series, J. Math. Anal. Appl. 179 (1993), no. 2, 646-649. MR 95a:42002. Zbl 797.42005.

[5] L. S. Bosanquet, A mean value theorem, J. London Math. Soc. 16 (1941), 146-148. MR 3,144e. Zbl 028.21901.

[6] T. M. Flett, On an extension of absolute summability and some theorems of Littlewood and Paley, Proc. London Math. Soc. (3) 7 (1957), 113-141. MR 19,266a. Zbl 109.04402. 
[7] _ Some more theorems concerning the absolute summability of Fourier series and power series, Proc. London Math. Soc. (3) 8 (1958), 357-387. MR 21\#1481. Zbl 109.04502.

[8] G. H. Hardy, Divergent Series, Clarendon Press, Oxford, 1949. MR 11:25a. Zbl 032.05801.

[9] S. M. Mazhar, On generalized quasi-convex sequence and its applications, Indian J. Pure Appl. Math. 8 (1977), no. 7, 784-790. MR 58\#29572. Zbl 415.42008.

[10] T. Pati, The summability factors of infinite series, Duke Math. J. 21 (1954), 271-283. MR 15,950e. Zbl 057.30002.

HÜSEYIN BOR: DEPARTMENT OF MATHEMATICS, ERCIYES UNIVERSITY 38039, KAYSERI, TURKEY E-mail address: bor@erciyes.edu.tr 


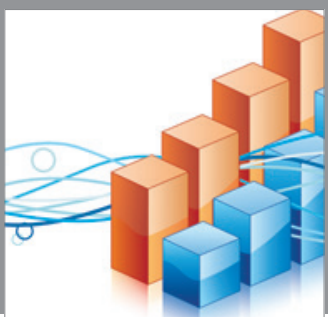

Advances in

Operations Research

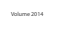

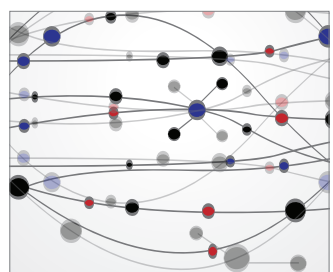

\section{The Scientific} World Journal
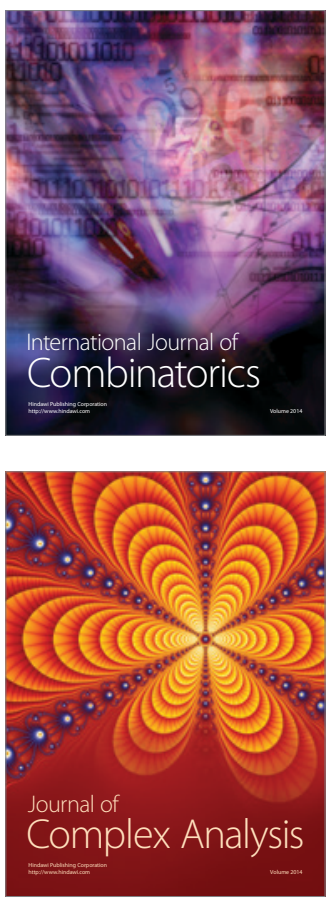

International Journal of

Mathematics and

Mathematical

Sciences
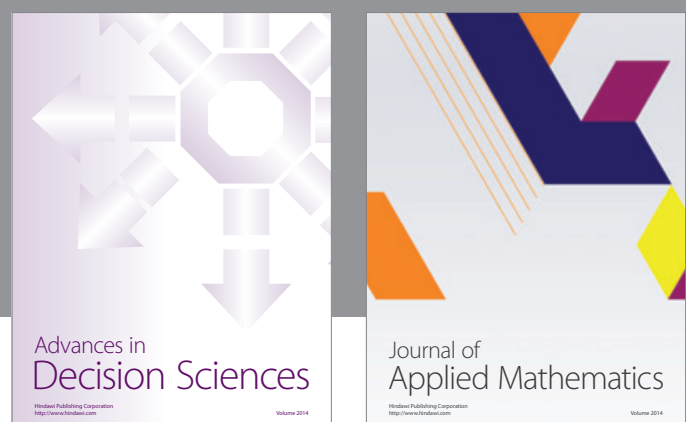

Journal of

Applied Mathematics
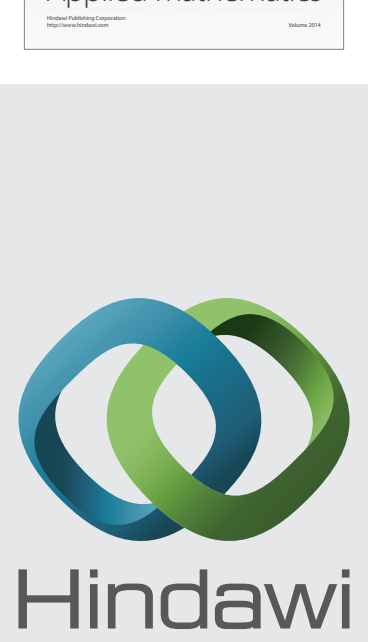

Submit your manuscripts at http://www.hindawi.com
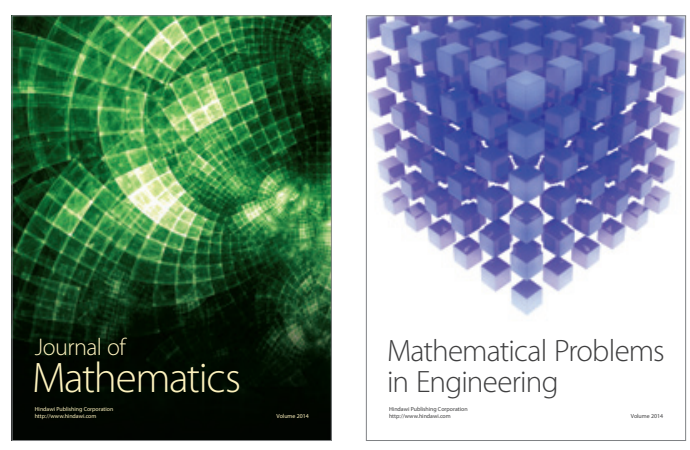

Mathematical Problems in Engineering
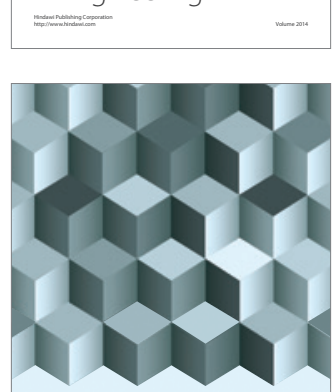

Journal of

Function Spaces
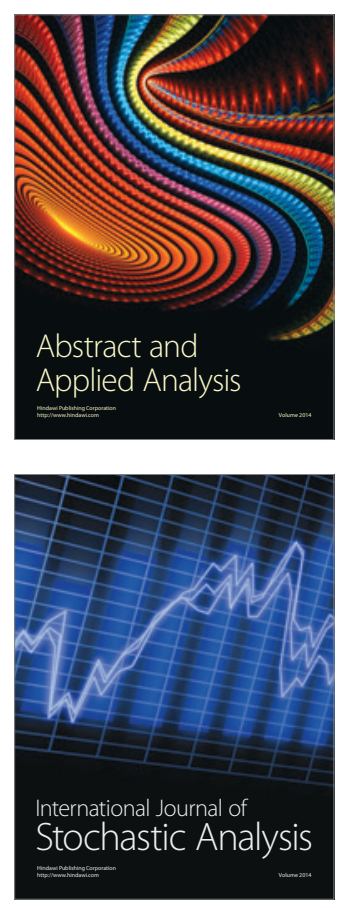

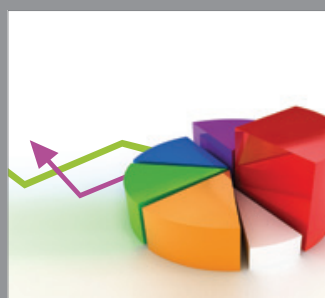

ournal of

Probability and Statistics

Promensencen
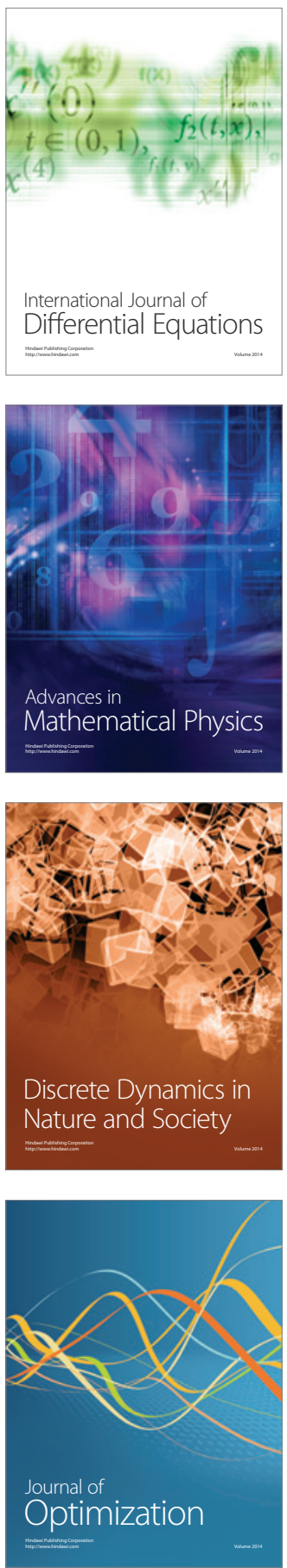